\title{
FATIGUE FRACTURE OF THE FIBULA IN CHILDHOOD
}

\author{
BY \\ ALAN L. GRIFFITHS \\ From the Royal Belfast Hospital for Sick Children
}

(RECEIVED FOR PUBLICATION MAY 3, 1952)

Fatigue, or stress, fractures have been described as occurring in many situations and at widely differing ages from 15 months to 64 years. Most have occurred in young adults, particularly soldiers in training and athletes. Although fatigue fractures usually occur in weight-bearing bones, in particular the metatarsals and tibia, they also occur in the fibula, and have even been described in the first rib and in the ulna.

The distinction between fatigue fractures and all other varieties of fracture arising in the absence of direct trauma has been clearly made by Blair Hartley (1943). Jackson Burrows (1948) in a comprehensive article, reviewed the literature of fatigue fracture of the fibula. In it he discussed the relationship between intensity and duration of stress. He described how stress of adequate intensity acting once produces a traumatic fracture, whereas a recurrent stress of much smaller intensity produces a fatigue fracture, and suggested that types intermediate between these extremes also occurred.

Fatigue fractures have been reported in several situations in children. Roberts and Vogt (1939) described a series of tibial fractures, the youngest child being 4 years old. Blair Hartley's (1942) series also included several children with tibial fractures. 'March' fractures also occur in children. Three cases were reported recently by Macpherson (1951), and during the year in which I collected these fibular fractures I saw six metatarsal fractures in five patients of ages ranging from 5 to $10 \frac{1}{2}$ years.

Siemens (1942) described a case of bilateral fatigue fracture of the middle third of each fibula in a boy of 15 months. Ingersoll (1943) described three cases in boys of 9 years, ascribing the lesion to the stress of learning ice skating. Fractures in one of his cases were bilateral. Siemens quotes Brandt as having done a biopsy on a girl of 10 years with a periosteal bony thickening above the lateral malleolus. In the absence of a history of injury he considered the case to be in the nature of a stress fracture.
The following eight cases were also in children, and show a number of points which may throw a little light on this interesting condition.

\section{Case Reports}

Case 1. Joseph M., aged $4 \frac{1}{2}$, was brought to the hospital on July 20,1950 , because he had been limping with his left leg for the previous three weeks. He would not admit to having sustained any injury. Tenderness over the lower third of the left fibula and pain on everting the foot were found on examination. The absence of fever, malaise or muscle tenderness was noted. A radiograph showed a transverse band of increased density extending across the fibula just below the junction of the middle and lower thirds. A narrow line of diminished density was present in the centre of this band on the lateral and posterior aspects. It extended out into a cloud of callus which surrounded the bone at this level and spread up and down the shaft. The amount of callus was greatest on the lateral side, where it tended to fill up the concavity of the bone. This was considered to be a 'greenstick' fracture of the fibula, and was treated by immobilization in a short leg plaster for a month. A second radiograph on August 3, taken through the plaster, showed a considerable increase in the amount of callus.

He did not attend after removal of the plaster until February 7, 1951, when he was brought up on account of a limp of five days' duration. This time the right leg was affected but once more there was no history of injury. He was tender over the fibula, and apyrexial. No bony changes were seen in the wet film of the fibula; so he was thought possibly to have sustained a bruise and treatment was not considered necessary. He returned nine days later, and was found to be exquisitely tender over the lower right fibula, but still apyrexial. The radiographs of the left fibula taken seven months previously were now seen, and the dissimilarity from a greenstick fracture remarked. A suspicion that the earlier fracture had been a fatigue fracture and that he now had a similar lesion in the other leg was supported by re-examination of the film taken nine days previously. On close inspection a small ' crack' could be seen in the lateral and posterior aspects of the right fibula in the upper part of its lower third. This was most clearly seen in the antero-posterior view, where a small haze of 


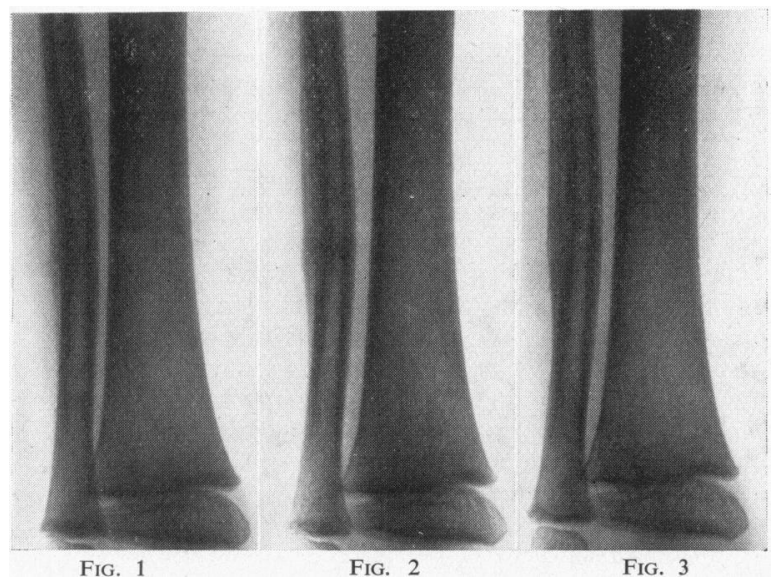

Fig. 1.-Right fibula two weeks after onset. Callus is now clearly visible (Case 1).

FIG. 2.-Right fibula four weeks after onset (Case 1)

FIG. 3.-Right fibula eight weeks after onset (Case 1).

callus was visible to the lateral side of the bone, with a central clear area opposite the crack in the cortex. A very faint band of increased density could be seen extending across the bone at this level. A new radiograph of this leg now showed a considerable increase in callus formation on the lateral concavity of the bone extending beyond its anterior and posterior margins, with a small central translucent area corresponding to the gap previously seen. The transverse band of sclerosis was more prominent, extending upwards as it ran forwards and medially. A short leg plaster was applied, and removed after a month. Because the child complained of pain it had to be re-applied a week later for a further 10 days. Serum calcium and phosphorus levels were normal; serum alkaline phosphatase was 20 KingArmstrong units. The leg was radiographed at intervals. The callus increased in amount and density. The band of sclerosis first increased in density and then gradually disappeared. The 'crack' became broader and then assumed an irregular cystic appearance before disappearing. Finally on May 3 the bone appeared normal apart from residual thickening of the cortex on the lateral side. The fibula remained slightly tender for about three months until this date, but this is perhaps explained by his conduct.

He was a particularly robust, healthy-looking child. His mother told me that he was a very active boy, who had been doing a lot of jumping in emulation of a strip cartoon character called 'Superman'. This took the form of jumping off stairs and furniture with a cloak around his shoulders. Moreover, this had continued even after immobilization in plaster, and accounted for the badly damaged state of his plasters which had required repair or renewal at each attendance. He had rather mobile ankle joints, with one inch separation of the malleoli when the knees were together. His shoes were badly worn on the inner side of the heels.

Case 2. Patsy $H_{\text {., }}$ aged 5 years and 2 months, was referred to us on March 30, 1951, on account of a swelling at the junction of the middle and lower thirds of the right fibula. She gave a history of pain and a limp since being kicked on the outer side of the leg by a little girl two weeks previously. Examination showed a tender swelling over the lower right fibula. A radiograph showed a cuff of callus surrounding the upper part of the lower third of the bone. This was heaped up on the lateral aspect opposite a broad, poorly defined area of slightly denser bone containing a central transverse line of rarefaction. A short leg plaster was applied. When removed on April 17 some tenderness aver the fibula was still present. A radiograph now showed that the callus had grown denser and the line of rarefaction more conspicuous. The resemblance between the $x$-ray appearances of this and the previous case was now remarked, and this was thought to be another fatigue fracture.

Her shoes were found to be worn to the uppers at the inner side of the heel. When questioned about unusual activity, her mother informed us that the child was learning to skip, and spent a lot of time jumping up and down.

At this attendance, four and a half weeks after the onset of symptoms, the blood calcium, phosphorus and phosphatase levels were found to be normal.

A viscopaste bandage was applied, and she was seen again a week later. Tenderness was still present, but she had continued to skip. When asked to jump, it was noticed that she did so with her feet flat, instead of springing from her toes. This flat-footed jump provoked careful observation of the local children, and it was realized that up to the age of 6 to 7 years most children jump in this way. She was still tender when seen again a fortnight later, so a $3 / 16$ in. inner raise of the heels of her shoes was prescribed. A radiograph on May 8 showed a greater density of callus. The zone of rarefaction had expanded to a broad band, with a zone of increased density on either side. She did not attend again.

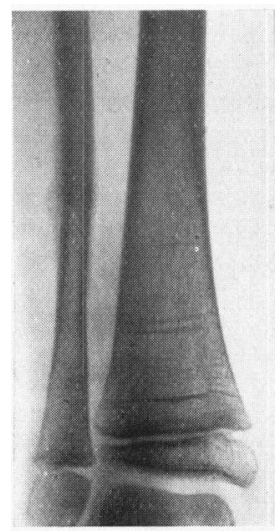

FIG 4.

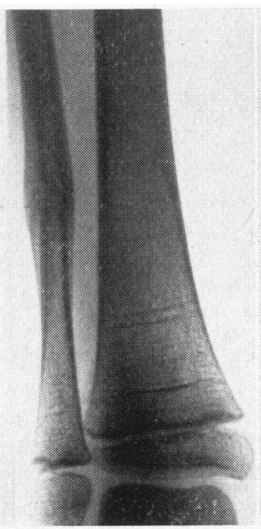

FIG. 5a

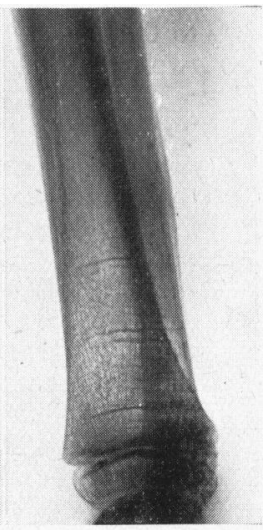

FIG. 5b
FIG. 4.-Right fibula. Antero-posterior view two weeks after onset (Case 2). Figs. 5 and 5a.-Right fibula : antero-posterior and lateral views eight weeks after onset (Case 2). 
Case 3. George L., aged 3 years and 4 months, was first seen on April 5, 1951, complaining of pain in his left leg and a limp following a fall two days before. He was tender over the lower fibula. A radiograph showed a narrow band of diminished density, running in an irregularly transverse line across the fibula towards the upper part of the lower third. This extended out into a cloud of callus surrounding the fibula, and had above and below it a well marked zone of increased density which also extended across the callus. This was diagnosed at the time as a healing fracture, unrelated to the recent injury, and no treatment was advised. When seen again five weeks later he was reported to have limped for a fortnight but had had no trouble since. A radiograph on May 11 showed a dense layer of callus surrounding the bone. The transverse zone of translucency no longer extended into the callus, and was wider and more irregular. The zones of sclerosis had almost disappeared. It was now realized that this was another fatigue fracture. No abnormality of feet or legs was found apart from some laxity of the ankle joints. He was wearing a pair of flat sandals too large for him, but at the relevant time had been wearing a pair of worn boots. Unfortunately, his mother could not remember on which side they had worn.

He had always had adequate cod liver oil and orange juice.

This was another child who played 'Superman', jumping off the furniture with a cloak around his shoulders.

Case 4. Unfortunately the notes on Jim B., aged 5 years, are scanty, and I did not see him personally at any time as he did not return after his first visit. He was sent up to be seen on April 20, 1951, on account of pain and tenderness over the lower end of the left fibula. There was no history of injury. A radiograph showed a faint sleeve of callus surrounding the fibula obliquely in its lower third, higher on the antero-lateral aspect. A minute nick was just visible in the callus on the lateral aspect. He was considered to have a 'slight periostitis', and adhesive strapping was applied to the ankle. The patient was told to return in one or two weeks, but did not attend.

Case 5. Barbara D., aged 5, first attended on April 21, 1951, with a history of pain over the outer side of the left leg of two weeks' duration, sufficiently severe to make her limp at times. She could not remember any injury. There was tenderness over the junction of the middle and lower thirds of the fibula. A radiograph showed a faint transverse line of diminished density running across the fibula in the lower part of its middle third. On either side of this line the bone appeared slightly more dense. A small amount of callus was present on the posterior and lateral aspects of the bone at this level. This was recognized to be a stress fracture by Mr. J. A. W. Bingham. A viscopaste bandage was considered sufficient treatment. When seen again a fortnight later she had had no further pain so the bandage was removed. No abnormality of feet or ankles was found and no history of predisposing stress or injury could be obtained. The mother remembered that the shoes she had been wearing had been badly worn, but could not recall where. A radiograph on May 5 showed an accentuation of the changes seen previously. She was asked to re-attend bringing her worn shoes, but did not come.

Case 6. Margaret S., a little girl, aged 2 years and 2 months, was first seen on May 25, 1951. She had been pushed by an older child and had fallen off a nine-inch step, landing on her right hip on a tiled floor. That evening she began to limp. Two days later she complained of pain in the right lower leg and was brought to the hospital. There was tenderness over the lower right fibula. A radiograph showed a very small amount of callus extending along the lateral concavity of the fibula with a minute nick in it just above the junction of the middle and lower thirds. The shaft of the bone showed no abnormality even when magnified. A short leg walking plaster was applied for six weeks. A radiograph on July 6 showed a considerable increase in the amount and density of callus, and an irregular oblique line of decalcification crossing the bone. The child had been wearing shoes with a built-in inner raise only slightly worn on the postero-lateral aspect of the heels. There was no history of jumping, skipping, or other stress. Adequate amounts of cod liver oil and orange juice had been taken. She was seen again on October 3 complaining of poorly localized pain in the other leg and foot of two days' duration. There was no tenderness and a radiograph showed no abnormality in the leg or foot. Three weeks later she was reported to have had no further pain but was not radiographed.

Case 7. Elizabeth McA., aged 8 years, attended on July 16 complaining of pain in the left leg. Twentyfour days previously a motor cycle had fallen on the outer side of her left leg causing considerable bruising. She had had pain causing a limp which had eased somewhat after two days and then remained steady. Examination showed a very tender swelling extending for four inches over the lower third of the leg on the outer side. There was pain on forced inversion but none on eversion. A radiograph showed a faint layer of callus to the lateral side of the lower third of the fibula and an even fainter cloud on the anterior aspect lower down. The callus appeared to be in two layers with a slight notch in the outer one. The fibula itself showed a rather doubtful suggestion of a very oblique line of translucency. This child had always been awkward on her feet, so that her father had nicknamed her 'Feet'. She had been skipping a lot before the accident and her mother had noticed that she jumped flat-footed. Her shoes were not badly worn. She was still tender when seen a week later, but was limping less. She did not attend again and no further radiographs were obtained.

The following case was found when checking the $x$-ray reports for the year. I did not see the case.

Case 8. Maeve R., aged $3 \frac{1}{2}$ years, fell and injured her left ankle. She attended hospital a week later on July 5,1950 , and was found to have marked generalized tenderness but no swelling. A radiograph (only one view available) showed a transverse crack extending 
through a deposit of callus and into the fibula, $2 \frac{1}{8}$ in. from the lower end. The disproportionate amount of callus present at one week does not appear to have been commented upon.

\section{Differential Diagnosis}

The differential diagnosis between fatigue fractures in general and pathological and pseudo-fractures has been clearly stated by Blair Hartley (1943). The conditions found in children in which pseudo or pathological fractures occur are rickets, the coeliac group of diseases, renal rickets, osteogenesis imperfecta and fragilitas ossium, tumours and cysts of bone, and following severe osteomyelitis. In these the diagnosis should present no difficulty owing to the abnormal radiological appearance of the bone and the presence of systemic disease.

Acute osteomyelitis is unlikely to be mistaken for a fatigue fracture, for in the one case the patient is ill and febrile and in the other is healthy and apyrexial, even though the degree of local tenderness may be comparable (Case 1).

Low-grade osteomyelitis or periostitis is less easy and more important to differentiate, for several cases are reported which have had unnecessary operations for fatigue fracture. When the fracture line is visible running through callus or cortex or through both the diagnosis is obvious, providing it is recognized that the condition does occur. When it is absent there may be a faint transverse line of rarefaction with a faint zone of increased density on either side, or there may be a broader single band of sclerosis. There is fusiform laying down of callus both on the inner and outer surfaces of the cortex, often on one side of the bone only. In low-grade osteomyelitis the lesion in the cortex tends to appear rounded or oval rather than linear, and there is a tendency for the subperiosteal new bone to extend more widely in an irregular manner.

Differentiation from sarcoma is likely to be difficult only if the appearances of a stress fracture are not recognized, and in a very early case with minimal x-ray changes. The regular line of osteoporosis and the smooth outline of new bone with no spicule formation are against a sarcoma. A repeat radiograph after a week will accentuate this difference.

\section{Precipitating and Predisposing Factors}

Fatigue fractures appear to be precipitated by unaccustomed duration of a stress which may have been met with previously in normal life. The association of walking or marching with the metatarsal fracture is well known. Fractures of this type in femur, tibia and fibula have been associated with other aspects of military training (Bingham, 1945; Kitchin and Richmond, 1945; Richmond, 1945). Runners and jumpers appear to be prone to fibular lesions (McPhee and Franklin, 1946; Weaver and Francisco, 1940; Ronald, 1945; Asal, 1936). Other factors must also operate, however, for while not all people subjected to apparently identical stress develop lesions, a few seem especially prone. In them two or even three bones may successively display fractures. A number of predisposing factors have been recognized in relation to the metatarsal fractures such as metatarsus atavicus. My cases are too few to be significant, but some inferences may be drawn.

The precipitating factor in Cases 1, 2 and 3 was almost certainly persistent jumping. I suggest that the predisposing factor in Case 1 was his shoes, which were badly worn on the inner side of the heel. Delay in repairing these shoes had accentuated the results of faulty posture. The worn shoes may have been a major factor in Case 2 also, and possibly in Case 3. This wearing at the heel was equivalent to a half inch outer raise, so that even when standing, there was an eversion strain on the ankle. Older children normally jump so as to land on their toes, but up to the age of 5 or 6 years, and in some cases to a still later age, children land with their feet flat, taking much of their weight on the heels. Case 2 was seen to jump in this manner and Cases 1 and 3 probably did likewise. In these cases, therefore, a very considerable eversion strain must have been thrown on the fibulae. This mechanism, however, cannot apply in Case 6, where the inner raise in the shoes tended to cause an inversion strain. Professor H. A. Harris describes a rapid development of the calf muscles at 7 years of age. This would correspond with this change in method of jumping. Professor Harris also describes the evertors of the foot as having a period of rapid growth. This occurs at about 18 months of age and must exert new stresses on the fibula. This may have a bearing on Siemens's case in an infant of 15 months.

The fibulae of my cases appear more strongly curved than many of those illustrated by other observers. They show a lateral concavity with the apex of the curve in the upper part of the lower third or lower part of the middle third. This apex only sometimes appears to correspond with the site of the fracture. The curve is absent or even reversed in Ingersoll's cases, and is not marked in Siemens's case. Possible reasons for this appearance are: (1) The curve is accentuated by different positioning in the radiograph. (2) The curve is due to active or previous rickets; there is no activity as 
judged by the two blood phosphatase estimations, and the epiphyses appear normal, and in two of the cases it was specifically stated that adequate vitamins had been taken. (3) Some infants have strongly curved tibiae concave medially at birth which gradually straighten. This straightening could conceivably cause curvature of the fibulae with resultant weakness. The curve may be due to the more rapid growth of the fibula than of the tibia which occurs in later foetal life. This curve is not due to trauma; it is present in normal controls and in the normal leg of Case 6.

\section{The Role of Trauma}

I suspect that, paradoxically, trauma (as distinct from stress) may play a part in the appearance of some fatigue fractures. It appears that fatigue fractures of the fibula may be symptomless until a late stage, provided that the precipitating stress is not unduly great. Blair Hartley (1942) has suggested that this is the case in fatigue fracture of the tibia. The fracture, for example, may be discovered by radiographs of the sound limb where there has been a similar fracture of the other leg as in Ingersoll's (1943) first case and in Siemens's case. Attention may be drawn to the fracture by a minor injury producing unduly great or prolonged pain, just as small localized bone abscesses are occasionally found in the same way. It may be also that the limp associated with the minor injury throws added stress on the fracture line, causing pain.

Since five of my eight cases have a history of injury, I must try to prove that they were not traumatic fractures. In Cases 3 and 6 there was callus two days after injury implying a rate of callus formation which does not occur even in the newborn. Even in Case 8, where the fall was a week 'before a radiograph was taken, the amount of callus was excessive. It is doubtful if Case 2 would have waited two weeks before attending had she been kicked hard enough to produce the fracture. In Case 7, however, the callus is rather scanty at 24 days especially when compared with a traumatic fracture without displacement at this level in a child of the same age who produced much more callus by the fifteenth day, and was quite recovered in 23 days. I feel that the fatigue fracture of Case 7 may have resulted from the stresses caused by limping. This discussion is analogous to that of Blair Hartley when describing fatigue fracture of the tibia. He suggests that $x$-ray changes may occur without symptoms. Moreover, five of his 14 cases gave a history of injury. The frequency with which a healthy child sustains various injuries makes it easy for an anxious parent to find a traumatic antecedent for any lesion.

\section{Level of Fracture}

No cases . of fracture of the upper third of the fibula occur in this series. It would be interesting to know if the lesion occurs in this situation in children, for, as Jackson Burrows suggests, in adults it appears to be associated with jumping. In my first three cases, which seem clearly associated with jumping, the fracture is in the lower third at a comparable site with that in a case (not reported here) in which fracture was due to falling suddenly and landing on both feet. In the latter case a stress of high intensity and short duration produced a similar lesion to that in the other cases where a similar but recurrent stress was acting at less intensity over a longer period.

Fatigue fractures seem to occur rarely in the middle third of the fibula. This is the situation in two of my cases (5 and 6) but to group them separately would be arbitrary. It may prove more useful to group them as occurring in the third quarter of the fibula to separate them from the cases with fracture low down near the syndesmosis. However, any system of classification can only be arbitrary until the precise aetiology has been determined. Fractures of the lower third occur in two main groups as pointed out by Jackson Burrows. The first occurs at about the level of the tibiofibular syndesmosis principally in 'elderly hard pressed women', but also in some runners (Weaver and Francisco, 1940; and Ronald, 1945). In the second group the fractures are situated more proximally, frequently twice as far from the tip of the malleolus. It is to this latter group that my cases belong, as do Ingersoll's cases, also in children, but the majority of cases published are in young athletes.

The site of the fracture line and of maximum deposition of callus is on the lateral side of the bone in all my cases, and is mostly towards the posterior rather than the anterior aspect. It is difficult to judge from published photographs, but Ingersoll's second and third cases appear to show most callus medially. This difference in position is difficult to explain but may be related to the straighter fibulae in his cases, or to a greater part played by rotation than eversion stresses. In Siemens's case the callus appeared on the lateral side of the fibulae.

\section{Summary}

Eight cases of fatigue fracture of the fibula are described and compared with other cases in the 
literature. All my cases occurred between the ages of 2 years 2 months and 8 years.

It is suggested that strongly curved fibulae and badly worn shoes may predispose to the development of fatigue fractures in the fibula, and jumping and skipping act as precipitating stresses.

The site of fracture associated with jumping is at a different level in children from that found in young adults.

The diagnosis should not be difficult if the condition is known to occur.

I should like to thank Mr. H. P. Hall, Mr. J. A. W. Bingham, Mr. I. Fraser and Mr. J. S. Loughridge, surgeons of the Royal Belfast Hospital for Sick Children, for permission to publish their cases. I am also indebted to Mr. H. Jackson Burrows, Mr. I. D. Kitchin and Mr. D. A. Richmond for their assistance with the literature.

REFERENCES

Asal, W. (1936), Arch. klin. Chir., 186, 511.

Bingham, J. A. W. (1945). Lancet, 2, 13

Burrows, H. Jackson (1948). J. Bone Jt Surg., 30-B, 266.

Hartley, J. Blair (1942). Brit. J. Surg., 30, 9.

- (1943). Brit. J. Radiol., 16, 255.

Ingersoll, C. F. (1943). Amer. J. Roentgenol., 50, 469.

Kitchin, I. D. and Richmond, D. A. (1945). Brit. med. J., 2, 214

Macpherson, D. A. (1951). Ibid., 2, 339.

McPhee, H. R. and Franklin, C. M. (1946). J. Amer. med. Ass., 131, 574.

Richmond, D. A. (1945). Iancet, 1, 273.

Roberts, S. M. and Vogt, E. C. (1939). J. Bone Jt Surg., 21, 891.

Ronald, A. (1945). Brit. J. Surg., 33, 90.

Siemens, W. (1942). Z Zl. Chir., 691809.

Weaver, J. B. and Francisco, C. B. (1940). J. Bone Jt Surg., 22, 610. 